\title{
Preoperative statin treatment is associated with reduced postoperative mortality and morbidity in patients undergoing cardiac surgery: An 8-year retrospective cohort study
}

Leslie L. Clark, MS, a,b John S. Ikonomidis, MD, PhD, ${ }^{a}$ Fred A. Crawford, Jr, MD, ${ }^{a}$ Arthur Crumbley III, MD, ${ }^{a}$ John M. Kratz, MD, ${ }^{a}$ Martha R. Stroud, MS, ${ }^{a}$ Robert F. Woolson, PhD, James J. Bruce, MS, ${ }^{\text {b }}$ Joyce S. Nicholas, PhD, ${ }^{\text {b }}$ Daniel T. Lackland, DrPH, ${ }^{\mathrm{b}}$ Michael R. Zile, MD, ${ }^{\mathrm{c}}$ and Francis G. Spinale, MD, PhD ${ }^{\mathrm{a}}$

See related editorial on page 520 .
From the Division of Cardiothoracic Surgery, ${ }^{a}$ Department of Biostatistics, Bioinformatics, and Epidemiology, ${ }^{\mathrm{b}}$ and Division of Cardiology, ${ }^{\mathrm{c}}$ Medical University of South Carolina, Charleston, SC.

Supported by National Heart, Lung, and Blood Institute grant 5 R01 HL056603-07 and an unrestricted educational grant from Merck.

Received for publication June 1, 2005; revisions received Aug 26, 2005; accepted for publication Aug 30, 2005.

Address for reprints: Francis G. Spinale, $\mathrm{MD}, \mathrm{PhD}$, Cardiothoracic Surgery, 114 Doughty St, Room 625, Medical University of South Carolina, Charleston, SC 29403 (E-mail: wilburnm@musc.edu).

J Thorac Cardiovasc Surg 2006;131:679-85

$0022-5223 / \$ 32.00$

Copyright $\odot 2006$ by The American Association for Thoracic Surgery

doi:10.1016/j.jtcvs.2005.11.006
Background: Cardiac surgical procedures can be associated with significant morbidity and mortality. Recently, it has been recognized that statins might induce multiple biologic effects independent of lipid lowering that could potentially ameliorate adverse surgical outcomes. Accordingly, this study tested the central hypothesis that pretreatment with statins before cardiac surgery would reduce adverse postoperative surgical outcomes.

Methods: Demographic and outcomes data were collected retrospectively for 3829 patients admitted for planned cardiac surgery between February 1994 and December 2002. Statin pretreatment occurred in 1044 patients who were comparable with non-statin-pretreated $(n=2785)$ patients with regard to sex, race, and age. Primary outcomes examined included postoperative mortality (30-day) and a composite morbidity variable.

Results: The odds of experiencing 30-day mortality and morbidity were significantly less in the statin-pretreated group, with unadjusted odds ratios of 0.43 (95\% confidence interval [CI], 0.28-0.66) and 0.72 (95\% CI, 0.61-0.86), respectively. Risk-adjusted odds ratios for mortality and morbidity were 0.55 (95\% CI, 0.32-0.93) and 0.76 (95\% CI, 0.62-0.94), respectively, by using a logistic regression model and 0.51 (95\% CI, 0.27-0.94) and 0.71 (95\% CI, 0.55-0.92), respectively, in the propensity-matched model, demonstrating significant reductions in 30-day morbidity and mortality. In a subsample of patients undergoing valve-only surgery $(\mathrm{n}=$ 716), fewer valve-only patients treated with statins experienced mortality, although these results were not statistically significant $(1.96 \%$ vs $7.5 \%)$.

Conclusions: These findings indicate that statin pretreatment before cardiac surgery confers a protective effect with respect to postoperative outcomes.

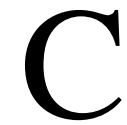

ommon cardiovascular surgical procedures include coronary artery bypass grafting (CABG), as well as valve repair and replacement. Although advancements in surgical technique and postoperative care have resulted in decreasing mortality and morbidity rates for these procedures, ${ }^{1,2}$ significant complications still occur. Thus identifying pretreatment strategies, such as pharmacologic interventions, that would favorably affect morbidity and mortality after surgical intervention remains an area of significant clinical interest.

A class of pharmacologic compounds that have stimulated significant interest with respect to the prevention of cardiovascular events are the 3-hydroxy-3methylglutaryl coenzyme A reductase inhibitors (statins). ${ }^{3-6}$ For example, the Heart Protection Study, a randomized placebo-controlled trial of more than 20,000 pa- 


\author{
Abbreviations and Acronyms \\ $\mathrm{CABG}=$ coronary artery bypass grafting \\ CI = confidence interval \\ LDL = low-density lipoprotein \\ MI = myocardial infarction
}

tients, conclusively demonstrated the ability of statins to reduce major cardiovascular events. Of note, this past study demonstrated that patients with normal baseline cholesterol levels also received as much benefit from statin therapy as those with increased low-density lipoprotein (LDL) cholesterol levels. In addition to other in vitro and in vivo studies, this report suggested that statins modify a number of cellular and extracellular processes that might be independent of direct lipid lowering. ${ }^{7,8}$ For instance, past studies have suggested that statins might have a beneficial effect on endothelial function in the context of hypertensive heart disease and diabetes. ${ }^{9-11}$ Other studies have suggested that statins might modify the release of biologically active molecules (eg, tumor necrosis factor $\alpha$ ) during and after myocardial ischemia. ${ }^{12}$ In light of these pleiotropic effects, it has been postulated that statin treatment might have beneficial effects in the context of cardiac surgery. ${ }^{13,14}$ Other studies have identified that statin treatment after CABG might favorably modify long-term morbidity and mortality. ${ }^{15,16}$ Accordingly, the present study was designed to test the central hypothesis that a significant risk reduction with respect to major early postoperative events would occur in patients receiving statin treatment preoperatively compared with those patients without statin treatment undergoing cardiac surgery. Although the penetrance of statin treatment in patients with coronary artery disease has increased over the past decade, a relatively high percentage of patients present for cardiac surgery without a prior history of statin treatment. In view of that, we conducted a retrospective study using logistic regression modeling and propensity score methodology to determine the effect of statin pretreatment on 30-day mortality and morbidity after cardiac surgery.

\section{Methods}

\section{Study Design}

Since 1993, all patients undergoing cardiac surgery at the Medical University of South Carolina have had their demographic and operative data entered into a registry. Preoperative use of statins in this cohort of surgical patients increased from less than $20 \%$ before 1997 to more than $60 \%$ in 2000 . The availability of these demographic, operative, and medication data allowed a retrospective evaluation of outcomes for patients treated with statins before surgical intervention versus those without while controlling for other potential confounding variables. This retrospective study was approved by the Institutional Review Board of the Medical University of South Carolina and conformed to all institutional review board and Health Insurance Portability and Accountability Act requirements.

\section{Patient Population}

Patients included in the cohort were 18 years of age or older and presented for nonemergency CABG, valve replacement, and/or valve repair surgery requiring cardiopulmonary bypass during the study period (1994-2002). Any patient presenting for operations involving congenital heart conditions were excluded from this analysis. The study cohort consisted of 3829 patients. Statin pretreatment occurred in 1044 patients.

\section{Study Data}

The surgery database used in this study was a complete patient database containing demographics, patient history, medical record information, intraoperative data, and postoperative outcome data. It mirrored the template for the current registry maintained nationally by the Society of Thoracic Surgeons. ${ }^{17}$ This surgical database was merged with the hospital pharmacy database in which all admission and discharge medications are recorded for each patient.

\section{Outcome Measures}

All outcomes were prespecified. Fatal and nonfatal outcomes were defined as follows: mortality occurring operatively or in the 30-day period postoperatively; myocardial infarction (MI); stroke or cerebral vascular event; hemodynamic collapse; reoperation; or a composite morbidity variable that included the occurrence of any of the morbidity outcomes. Outcomes were adjudicated by all attending cardiothoracic surgeons and intensivists at a weekly surgical morbidity and mortality conference.

\section{Variable Selection}

The variables analyzed included demographic variables; preoperative, intraoperative, and postoperative data; and medication data. Dichotomous and categoric variables included variables such as race and sex and variables indicating the type of surgical procedure (eg, isolated $\mathrm{CABG}, \mathrm{CABG}$ with valve repair, and isolated valve repair), as well as the presence or absence of comorbidities. Continuous variables measured were age and body surface area. Variable selection for the logistic model initially involved prespecification of the model by using clinician input for relevant covariates, as well as a literature review of predictive mortality and morbidity models in cardiac surgery. ${ }^{18-20}$ Variables selected for inclusion in the model included patient age, sex, race, preexisting comorbidities, type of cardiac procedure, previous cardiac surgery, ejection fraction, and New York Heart Association class. In addition, predictor variables that were significant at a 2 -tailed $P$ value of less than .15 in univariate analyses were entered into the full model.

\section{Data Analysis}

Baseline demographics for patients who received statin treatment were compared with those of patients who had not received statin treatment. $\chi^{2}$ Tests were used to determine differences among treated and nontreated patients for categoric variables. The Wilcoxon rank sum test and $t$ tests were used to compare the 2 groups for continuous variables. Complete data were available on out- 
TABLE 1. Statin use in the statin-treated group

\begin{tabular}{cc}
\hline Type and dose of statin & No. treated (\%) \\
\hline Atorvastatin & \\
$10 \mathrm{mg}$ & $252(24.14)$ \\
$20 \mathrm{mg}$ & $114(10.92)$ \\
$40 \mathrm{mg}$ & $52(5.00)$ \\
Simvastatin & \\
$10 \mathrm{mg}$ & $210(20.11)$ \\
$20 \mathrm{mg}$ & $238(22.81)$ \\
$60 \mathrm{mg}$ & $19(1.82)$ \\
Lovastatin & \\
$20 \mathrm{mg}$ & $60(5.75)$ \\
$40 \mathrm{mg}$ & $11(1.05)$ \\
Fluvastatin & \\
$20 \mathrm{mg}$ & $25(2.39)$ \\
$40 \mathrm{mg}$ & $5(0.48)$ \\
Pravastatin & \\
$10 \mathrm{mg}$ & $4(0.38)$ \\
$20 \mathrm{mg}$ & $41(3.93)$ \\
$40 \mathrm{mg}$ & $13(1.25)$ \\
\hline
\end{tabular}

come variables and statin use for all patients. Complete covariate data were available for 3409 patients.

The unadjusted effects of statin use on 30-day morbidity and mortality were assessed by using logistic regression analysis. Riskadjusted comparisons were made by using multivariable logistic regression models after adjustment for preoperative patient risk factors. In addition, a second method of adjustment using matching on propensity scores was used. Conditional logistic regression was used to analyze these matched pairs and determine the effect of statin pretreatment.

\section{Propensity Score Development}

This study followed a retrospective and nonrandomized design. Therefore there was no control over which patients received statin treatment before surgical intervention versus those who did not. Issues of selection bias and confounding were addressed through the use of a propensity score, a statistical strategy introduced by Rubin and Rosenbaum as a means of addressing the bias that exists in observational studies. ${ }^{21,22}$ Multiple logistic regression was used in this study to construct propensity scores for statin use. Patients from the statin-treated and statin-untreated groups were then matched on propensity scores, and logistic regression was used to determine the effect of statin treatment on morbidity and mortality. After matching on propensity score, there were no significant differences between the 2 groups.

\section{Results}

The study population was drawn from patients undergoing elective cardiac surgery during the period from 1994 through 2002. The use of preoperative statins increased from less than $20 \%$ in the initial year of this study to more than $60 \%$ in the final year of this study $(P<.001$ for time trend).

Table 1 delineates the type and dose of statin in the statin-treated group. No differences in mortality or morbidity outcomes were observed by type of statin used.

Table 2 presents the overall clinical characteristics for patients treated and not treated with statins. Age and sex distributions were similar in both groups. Body surface area was significantly greater in the statin-treated group. This group was also more likely to have had a previous $\mathrm{MI}(P=$ $.0007)$ or to be diabetic $(P<.002)$, hypertensive $(P<$ $.001)$, or hypercholesterolemic $(P<.001)$.

TABLE 2. Baseline patient characteristics

\begin{tabular}{|c|c|c|c|}
\hline Variable & $\begin{array}{l}\text { Not treated } \\
(n=2785)\end{array}$ & $\begin{array}{c}\text { Statin treated } \\
(\mathrm{n}=1044)\end{array}$ & $P$ value \\
\hline \multicolumn{4}{|l|}{ Demographic } \\
\hline Age & $63.39 \pm 12.55$ & $63.18 \pm 11.2$ & .64 \\
\hline Body surface area & $1.93 \pm 0.33$ & $1.96 \pm 0.22$ & .007 \\
\hline Male, \% & 66.5 & 66.85 & 0.836 \\
\hline \multicolumn{4}{|l|}{ Risk factors-comorbidities } \\
\hline Current smoker, \% & 58.71 & 58.27 & .805 \\
\hline Hypertensive, \% & 67.02 & 75.78 & $<.001$ \\
\hline Diabetes, $\%$ & 28.32 & 33.33 & .002 \\
\hline Hypercholesterolemia, \% & 37.95 & 79.79 & $<.001$ \\
\hline Renal failure, $\%$ & 6.78 & 6.63 & .865 \\
\hline NYHA class IV, $\%$ & 34.53 & 32.06 & .143 \\
\hline Previous MI, \% & 37.32 & 52.81 & .000 \\
\hline Cerebrovascular disease, $\%$ & 13.91 & 15.24 & .293 \\
\hline Peripheral vascular disease, \% & 15.42 & 15.64 & .863 \\
\hline Immunosuppressive treatment, \% & 2.09 & 2.12 & .968 \\
\hline Preoperative use of intra-aortic balloon pump, $\%$ & 3.48 & 2.9 & .264 \\
\hline
\end{tabular}

NYHA, New York Heart Association; MI, myocardial infarction. 
TABLE 3. Use of preoperative medications

\begin{tabular}{lccr}
\hline Variable & $\begin{array}{c}\text { Not treated } \\
(\mathbf{n}=\mathbf{2 7 8 5})\end{array}$ & $\begin{array}{c}\text { Statin treated } \\
(\mathbf{n}=\mathbf{1 0 4 4 )}\end{array}$ & $\boldsymbol{P}$ value \\
\hline $\begin{array}{l}\beta \text {-Adrenergic blockers, \% } \\
\text { Digitalis, \% }\end{array}$ & 44.11 & 61.69 & $<.001$ \\
Calcium antagonists, \% & 18.87 & 11.57 & $<.001$ \\
$\begin{array}{l}\text { Angiotensin-converting } \\
\quad \text { enzyme inhibitors, \% }\end{array}$ & 28.98 & 32.02 & .067 \\
Oral nitrates, \% & 28.08 & 32.05 & .016 \\
Use of diuretics, \% & 38.07 & 55.11 & $<.001$ \\
Use of aspirin, \% & 35.01 & 29.45 & .001 \\
\hline
\end{tabular}

Use of other medications before surgical intervention also differed between the 2 groups. The statin-treated group had a higher percentage of patients treated with $\beta$-adrenergic blockers $(P<.001)$, angiotensin-converting enzyme inhibitors $(P=.016)$, oral nitrates $(P<.001)$, and aspirin $(P<$ $.001)$. Those not treated with statins had a higher incidence of digitalis $(P<.001)$ or diuretic use $(P=.001$, Table 3$)$.

The percentage of patients experiencing 30-day morbidity was significantly less in the statin-treated group (5.9\% vs $8.3 \%, P<.05)$. Statin-treated patients also experienced lower mortality in the 30 -day period $(2.5 \%$ vs $5.6 \%, P<$ .05; Figure 1).

Risk-adjusted outcomes also demonstrated that statintreated patients experienced lower odds of operative mortality and morbidity. This was true for the standard riskadjusted model (adjusted for age, sex, body surface area, smoking, previous MI, cerebrovascular and peripheral vascular disease, diabetes, ejection fraction, New York Heart Association functional class, hypertension, $\beta$-adrenergic blocker use, aspirin use, use of intra-aortic balloon pump, and operative category), with an odds ratio of 0.55 (95\% confidence

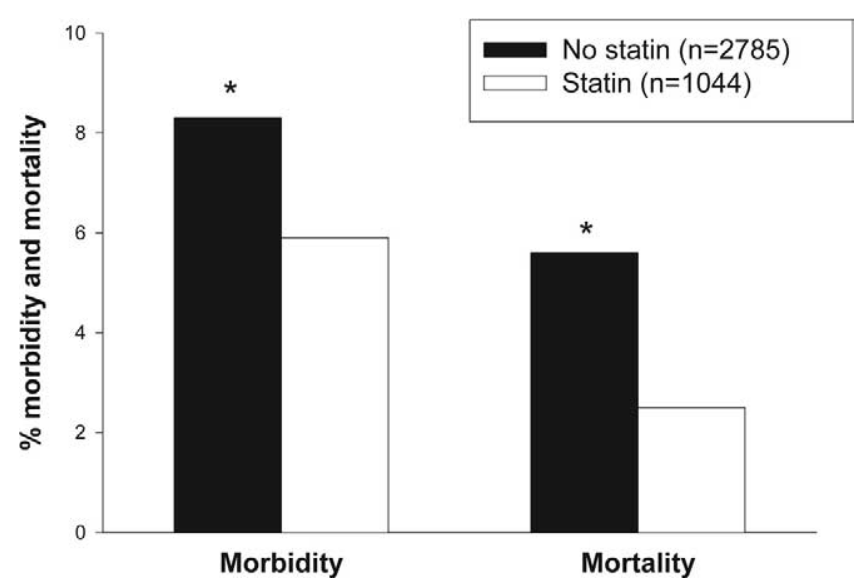

Figure 1. Unadjusted percentages of 30-day morbidity and mortality for patients pretreated with statins $(n=1044)$ or not treated (n $=2785$ ). ${ }^{*} P<.05$ versus not treated. interval [CI], 0.32-0.93) for mortality and 0.76 (95\% CI, 0.62-0.94) for morbidity. Similar results were seen after matching patients on treatment propensity scores, with an odds ratio of 0.51 (95\% CI, 0.27-0.94) for mortality and 0.71 (95\% CI, 0.5500.92) for morbidity (Figure 2). Primary cause of death for both statin treated and not treated groups are shown in Figure 3.

$\mathrm{CABG}$ or $\mathrm{CABG}$ with valve procedures accounted for approximately $80 \%$ of the sample $(\mathrm{n}=3113)$, whereas $20 \%$ underwent valve repair or replacement only $(\mathrm{n}=716)$. Univariate analysis demonstrated that 30-day mortality was higher in the valve-only group $(7.5 \%$ vs $2.0 \%, P<.001)$. Statin use was less common in the valve-only group (7\% vs $32 \%, P=.002)$. This necessitated controlling for operative category in the risk-adjusted analysis. In addition, the percentage of valve-only patients experiencing mortality was lower for statin-treated patients $(1.96 \%$ vs $7.5 \%)$. This was not statistically significant, likely a result of the reduction in power caused by the reduced sample size.

\section{Discussion}

Despite advances in surgical techniques, ${ }^{23,24}$ hemodynamic monitoring, and postoperative care, cardiac surgical procedures still incur significant morbidity and mortality. ${ }^{25}$ Furthermore, a complicated postoperative course will result in a significant medical cost burden. ${ }^{25}$ Accordingly, the development of strategies to further reduce morbidity and mortality associated with cardiac surgical procedures would be of significant clinical import. The 3-hydroxy-3-methylglutaryl coenzyme A reductase inhibitors (statins) have demonstrated efficacy in the reduction of cardiovascular events in primary and secondary prevention in varied patient populations, including surgical populations. ${ }^{3,5,6,15,16,26-31}$ The overall

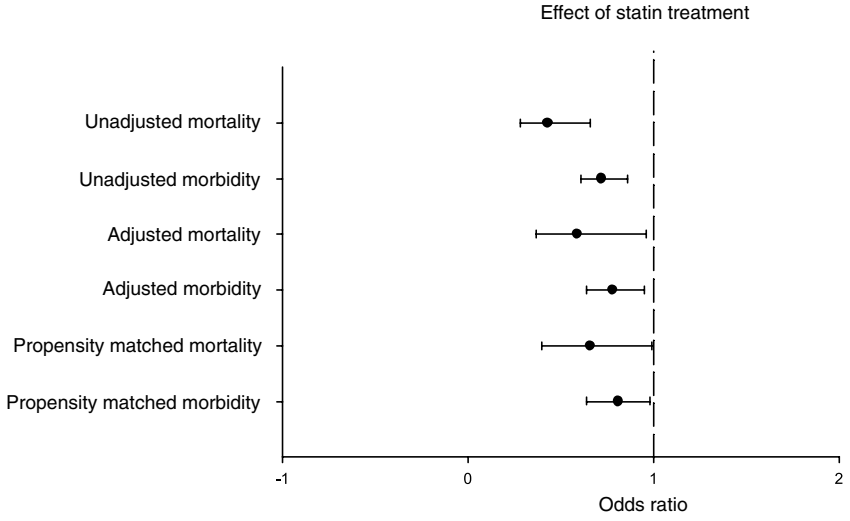

Figure 2. Comparison on odds ratios for unadjusted, adjusted, and propensity-matched models. The solid points represent the odds ratio, and the lines represent the $95 \%$ confidence interval. All of the odds ratios fall below the null value of 1 , indicating a significantly reduced risk of 30-day mortality and morbidity outcome for patients pretreated with statins $(P<.05$ vs not treated). 

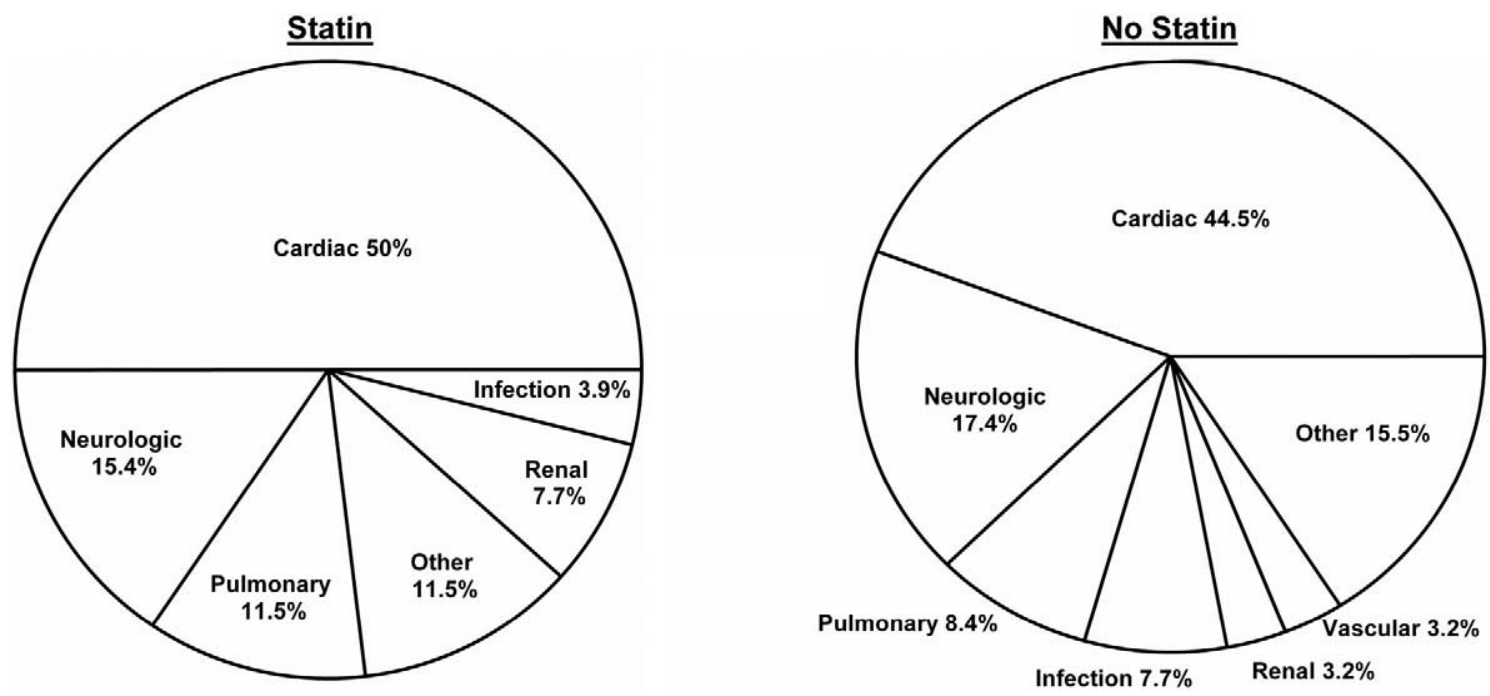

Figure 3. Comparison of primary cause of mortality in patients pretreated with statins (number of events $=26$ ) and not treated (number of events $=155$ ). Primary cause of mortality was defined as the body system that was the first significant system that began to fail or was the precipitating system that ultimately led to the patient's death. These systems were defined as follows: cardiac, myocardial infarction, arrhythmic disorder, cardiomyopathy, or heart failure; neurologic, stroke, cerebral bleed, embolism, or other neurologic event; pulmonary, respiratory insufficiency or failure, pneumonia, adult respiratory distress syndrome, ventilator dependence, or pulmonary edema; infection, any systemic or organ infection (ie, sepsis, sternal or wound, subacute bacterial endocarditis, or cellulitis); renal, renal failure, dialysis, or both; vascular, peripheral (arterial circulation) disorder, such as embolic event or circulatory dysfunction; other, cause other than those listed above, accidental, trauma, multiple organ failure, or other surgical intervention that led to patient's death.

goal of the present study was to evaluate whether statin pretreatment exerted an independent effect on 30-day morbidity and mortality in a cohort of patients undergoing cardiac procedures requiring cardiopulmonary bypass. The unique and important findings of this study were that preoperative statin use was independently associated with an approximately $40 \%$ reduction in 30-day mortality, and an effect of similar but lesser magnitude was observed with a composite morbidity measure encompassing postoperative MI, stroke, reoperation, renal failure, and infection. Consequently, this study provides a rationale and foundation for developing a pretreatment regimen for statin therapy in patients before cardiac surgery.

It is becoming increasingly clear that statins have pleiotropic effects independent of lipid lowering that could result in a reduction of adverse postoperative events. These effects include the ability to improve endothelial function, decrease vasoconstriction, suppress the inflammatory response, and reduce thrombosis. ${ }^{13,14,32}$ In the present study there was a high incidence of patients undergoing cardiac surgical procedures who had preexisting comorbid conditions, such as diabetes, hypertension, and hypercholesterolemia. Thus these patients have preexisting endothelial dysfunction, have increased levels of circulating inflammatory markers, and are more prone to vasoconstriction and thrombosis, which in turn results in these patients being at greater operative risk. Notably, the incidence of these comorbid conditions was significantly higher in the statin-pretreated group. However, despite the increased risk conferred by these concomitant disease states, the statin-pretreated group still experienced reduced acute morbidity and mortality. It has been established that statins exert cardioprotective effects in high-risk groups. For example, statins have demonstrated efficacy in reducing rates of cardiovascular events in diabetic patients, irrespective of their initial cholesterol levels. ${ }^{28}$ In addition, statins have been shown to exert even more powerful and positive effects on the endothelium in diabetic patients than in healthy control subjects. ${ }^{9}$ The present study was retrospective and therefore could not define the mechanisms behind the observed effects of statins on postsurgical morbidity and mortality. Nevertheless, the results of this study provide the fundamental basis for future studies designed to elucidate the possible mechanisms behind these effects.

Numerous large clinical trials have conclusively demonstrated that statin treatment reduces the risk of cardiovascular events for both primary and secondary prevention..$^{3-6,33}$ Both animal models and observational studies have shown that 
preoperative statin therapy can have beneficial effects in the perioperative period. ${ }^{29,31,34,35}$ In a porcine model pretreatment with atorvastatin for 3 weeks before CABG resulted in myocardial protection, as evidenced by decreased infarct size, improved regional wall motion, and preserved endothelial function in the statin-treated animals. These changes occurred irrespective of changes in cholesterol levels. ${ }^{35}$ In accordance with our findings, the most recent of the observational studies reported that preoperative statin treatment was associated with a significant reduction in 30-day mortality by using multivariate logistic regression analysis. ${ }^{34}$ Although this study did not replicate this result in its propensity score-matched analysis (likely because of a loss of statistical power as a result of the matching strategy), propensity-matched analysis did demonstrate a significant reduction in a composite variable comprised of 30-day mortality and stroke. In the present study our retrospective analysis comprises the largest single-institution study to date evaluating the effect of preoperative statin treatment on morbidity and mortality in patients undergoing cardiac surgery. Even after adjustment for numerous covariates, patients treated with statins before surgical intervention had greatly lower morbidity and mortality rates. Because of the much larger sample size in the present study, the results of both the multivariate logistic regression and propensity score-matched analysis show the same reduction in risk of 30-day mortality. Additionally, our data also show an approximately $25 \%$ reduction in a composite morbidity measure. In addition, unlike other studies, our patient population was not restricted to patients undergoing CABG but also included patients undergoing valve repair and replacement procedures. Although including these patients necessitated controlling for operative category in our analysis, this increases the generalizability of our findings and might also indicate that the effects of statin pretreatment might transcend the cardiac disease process and instead influence more intrinsic biologic processes. Because risk models for valvular heart disease are not as well developed as those for coronary disease, the risk adjustment strategies used might not adequately differentiate results between $\mathrm{CABG}, \mathrm{CABG}$ valve, and valve-only patients. In addition, our ability to investigate the effects of statin pretreatment in the valveonly group is limited by both reduced sample size and a limited number of events in the statin-treated valve-only group. However, analysis of the valve-only subsample was suggestive that valve-only patients also experienced a beneficial effect of statin pretreatment, and this result could form the basis of future studies.

The present study also detected differences in medication profiles between the 2 groups. Notably, the statin-pretreated group also took more $\beta$-adrenergic receptor antagonists, angiotensin-converting enzyme inhibitors, and aspirin. The increased use of these medications is likely due to the greater observed rate of previous MI observed in the statin-pretreated group. In large observational studies both $\beta$-adrenergic blockers ${ }^{36}$ and aspirin ${ }^{37}$ have demonstrated a survival benefit in patients undergoing CABG. Accordingly, we adjusted for the use of these medications in our analysis. Statin pretreatment still exhibited an independent association with reduced postsurgical morbidity and mortality. However, it is possible that the higher rates of cardioprotective medications seen in the statin-treated group might indicate these patients were better prepared for surgical intervention and received more preoperative care than the non-statin-treated group. It has been demonstrated that a patient cohort with increased use of the combination of statins, aspirin, and $\beta$-adrenergic receptor blockers after a coronary event had fewer recurrent MIs and lower rates of cardiac mortality. ${ }^{38}$ Future studies that model the potential additive effects, synergistic effects, or both of statins with other medications would be warranted.

Although the present study clearly demonstrated the positive effect of statin treatment, the necessary dosing regimen to establish these effects has yet to be established. A small observational study demonstrated that short-term statin therapy (3-5 days) preoperatively has been associated with reduced cardiovascular adverse events after $\mathrm{CABG} .{ }^{29}$ In addition, statins have shown rapid effects on both endothelial function and inflammatory markers, producing changes in as little as 24 hours to 1 week. ${ }^{10}$ Further studies are necessary to define the optimal temporal window for statin pretreatment before cardiac procedures. Another consideration in the present study is that although 2 statistical methods were used to control for potential confounding variables, there might be unmeasured covariates that are influencing the association seen between statin pretreatment and morbidity and mortality outcomes. Only prospective randomization can ensure (on average) that there are no systematic differences in covariates. We could not use that strategy because of the retrospective nature of this study. However, the propensity score methodology used allows a quasirandomization, which should result in unbiased estimates of the treatment effect. ${ }^{22}$

Two questions that remain unanswered by this study are whether the statin-treated group met target levels of LDL control and whether there were significant differences in LDL levels between the groups. If no significant differences existed, this would contribute further evidence that the demonstrated beneficial effects of statins in this study were due to pleiotropic effects. Unfortunately, preoperative levels of LDL were not recorded in each group, and thus whether a significant difference in LDL levels existed between the groups cannot be determined.

These limitations notwithstanding, the present study provides direct evidence that statin pretreatment before cardiac 
surgical procedures imparts a beneficial effect with regard to reduced rates of acute morbidity and mortality. Our data provide a foundation for prospective studies evaluating the optimal statin regimen to initiate before cardiac procedures.

\section{References}

1. Estafanous FG, Loop FD, Higgins TL, et al. Increased risk and decreased morbidity of coronary artery bypass grafting between 1986 and 1994. Ann Thorac Surg. 1998;65:383-9.

2. Aranki SF, Rizzo RJ, Adams DH, et al. Single-clamp technique: an important adjunct to myocardial and cerebral protection in coronary operations. Ann Thorac Surg. 1994;58:296-303.

3. Randomised trial of cholesterol lowering in 4444 patients with coronary heart disease: the Scandinavian Simvastatin Survival Study (4S). Lancet. 1994;344:1383-9.

4. The Long-Term Intervention with Pravastatin in Ischaemic Disease (LIPID) Study Group. Prevention of cardiovascular events and death with pravastatin in patients with coronary heart disease and a broad range of initial cholesterol levels. N Engl J Med. 1998;339:1349-57.

5. Shepherd J, Cobbe SM, Ford I, et al. Prevention of coronary heart disease with pravastatin in men with hypercholesterolemia. $N$ Engl J Med. 1995;333:1301-8.

6. MRC/BHF Heart Protection Study of cholesterol lowering with simvastatin in 20,536 high-risk individuals: a randomised placebocontrolled trial. Lancet. 2002;360:7-22.

7. Salam AM. Expanding indications of statins; implications of the Heart Protection Study. Expert Opin Investig Drugs. 2003;12:509-13.

8. McFarlane SI, Muniyappa R, Francisco R, Sowers JR. Pleiotropic effects of statins: lipid reduction and beyond. J Clin Endocrinol Metab. 2002;87:1451-8.

9. Economides PA, Caselli A, Tiani E, Khaodhiar L, Horton ES, Veves A. The effects of atorvastatin on endothelial function in diabetic patients and subjects at risk for type 2 diabetes. J Clin Endocrinol Metab. 2004;89:740-7.

10. Wassmann S, Nickenig G. Improvement of endothelial function by HMG-CoA reductase inhibitors. Drug News Perspect. 2002;15:85-92.

11. Wassmann S, Laufs U, Muller K, et al. Cellular antioxidant effects of atorvastatin in vitro and in vivo. Arterioscler Thromb Vasc Biol. 2002;22:300-5.

12. Kinlay S, Schwartz GG, Olsson AG, et al. High-dose atorvastatin enhances the decline in inflammatory markers in patients with acute coronary syndromes in the MIRACL study. Circulation. 2003;108: 1560-6.

13. Lazar HL. Role of statin therapy in the coronary bypass patient. Ann Thorac Surg. 2004;78:730-40.

14. Werba JP, Tremoli E, Massironi P, et al. Statins in coronary bypass surgery: rationale and clinical use. Ann Thorac Surg. 2003;76:2132-40.

15. The Post Coronary Artery Bypass Graft Trial Investigators. The effect of aggressive lowering of low-density lipoprotein cholesterol levels and low-dose anticoagulation on obstructive changes in saphenousvein coronary-artery bypass grafts. $N$ Engl J Med. 1997;336:153-62.

16. Sacks FM, Pfeffer MA, Moye LA, et al. The effect of pravastatin on coronary events after myocardial infarction in patients with average cholesterol levels. Cholesterol and Recurrent Events Trial investigators. N Engl J Med. 1996;335:1001-9.

17. Grover FL, Shroyer AL, Hammermeister K, et al. A decade's experience with quality improvement in cardiac surgery using the Veterans Affairs and Society of Thoracic Surgeons national databases. Ann Surg. 2001;234:464-74.

18. Gardner SC, Grunwald GK, Rumsfeld JS, et al. Comparison of shortterm mortality risk factors for valve replacement versus coronary artery bypass graft surgery. Ann Thorac Surg. 2004;77:549-56.

19. Huang CH, Lai ST, Weng ZC. Risk factors for mortality in primary isolated coronary artery bypass grafting surgery. J Formos Med Assoc. 2001;100:299-303.
20. Shroyer ALW, Coombs LP, Peterson ED, et al. The society of thoracic surgeons: 30-day operative mortality and morbidity risk models. Ann Thorac Surg. 2003;75:1856-65.

21. Rosenbaum P, Rubin D. Reducing bias in observational studies using subclassification on the propensity score. J Am Stat Assoc. 1984;79: 516-24.

22. D'Agostino RB Jr. Propensity score methods for bias reduction in the comparison of a treatment to a non-randomized control group. Stat Med. 1998;17:2265-81.

23. Loop FD, Irarrazaval MJ, Bredee JJ, Siegel W, Taylor PC, Sheldon WC. Internal mammary artery graft for ischemic heart disease. Effect of revascularization on clinical status and survival. Am J Cardiol. 1977;39:516-22.

24. Lichtenstein SV, Ashe KA, el Dalati H, Cusimano RJ, Panos A, Slutsky AS. Warm heart surgery. J Thorac Cardiovasc Surg. 1991; 101:269-74.

25. Mangano DT. Cardiovascular morbidity and CABG surgery-a perspective: epidemiology, costs, and potential therapeutic solutions. J Card Surg. 1995;10:366-8.

26. Alaupovic P, Fesmire JD, Hunnighake D, et al. The effect of aggressive and moderate lowering of LDL-cholesterol and low dose anticoagulation on plasma lipids, apolipoproteins and lipoprotein families in post coronary artery bypass graft trial. Atherosclerosis. 1999;146: 369-79.

27. Albert MA, Danielson E, Rifai N, Ridker PM. Effect of statin therapy on C-reactive protein levels: the pravastatin inflammation/CRP evaluation (PRINCE): a randomized trial and cohort study. JAMA. 2001; 286:64-70.

28. Collins R, Armitage J, Parish S, Sleigh P, Peto R. MRC/BHF Heart Protection Study of cholesterol-lowering with simvastatin in 5963 people with diabetes: a randomised placebo-controlled trial. Lancet. 2003;361:2005-16.

29. Dotani MI, Elnicki DM, Jain AC, Gibson CM. Effect of preoperative statin therapy and cardiac outcomes after coronary artery bypass grafting. Am J Cardiol. 2000;86:1128-30.

30. Sever PS, Dahlof B, Poulter NR, et al. Prevention of coronary and stroke events with atorvastatin in hypertensive patients who have average or lower-than-average cholesterol concentrations, in the Anglo-Scandinavian Cardiac Outcomes Trial-Lipid Lowering Arm (ASCOT-LLA): a multicentre randomised controlled trial. Lancet. 2003;361:1149-58.

31. Poldermans D, Bax JJ, Kertai MD, et al. Statins are associated with a reduced incidence of perioperative mortality in patients undergoing major noncardiac vascular surgery. Circulation. 2003;107:1848-51.

32. Davignon J. Beneficial cardiovascular pleiotropic effects of statins. Circulation. 2004;109(suppl III):III39-43.

33. Spencer FA, Allegrone J, Goldberg RJ, et al. Association of statin therapy with outcomes of acute coronary syndromes: the GRACE Study. Ann Intern Med. 2004;140:857-66.

34. Pan W, Pintar T, Anton J, Lee VV, Vaughn WK, Collard CD. Statins are associated with a reduced incidence of perioperative mortality after coronary artery bypass graft surgery. Circulation. 2004;110(suppl II):II45-9.

35. Lazar HL, Bao Y, Zhang Y, Bernard SA. Pretreatment with statins enhances myocardial protection during coronary revascularization. J Thorac Cardiovasc Surg. 2003;125:1037-42.

36. Ferguson TB, Jr, Coombs LP, Peterson ED, for the Society of Thoracic Surgeons National Adult Cardiac Surgery Database. Preoperative $\beta$-blocker use and mortality and morbidity following CABG surgery in North America. JAMA. 2002;287:2221-7.

37. Mangano DT, the Multicenter Study of Perioperative Ischemia Research Group. Aspirin and mortality from coronary bypass surgery. N Engl J Med. 2002;347:1309-17.

38. Fonarow GC, Gawlinski A, Moughrabi S, Tillisch JH. Improved treatment of coronary heart disease by implementation of a Cardiac Hospitalization Atherosclerosis Management Program (CHAMP). Am J Cardiol. 2001;87:819-22. 